\title{
ANÁLISIS DEL PLAN DE ATENCIÓN A LA DIVERSIDAD DE UN CENTRO EDUCATIVO. ESTUDIO DE CASO.
}

\author{
Efrén Labernia JIMÉNEZ ${ }^{1}$ \\ Jorge Ortega GONZÁLEZ ${ }^{2}$ \\ Eladio Sebastián HEREDERO ${ }^{3}$
}

\begin{abstract}
RESUMEN: Esta investigación recoge un estudio del Plan de Atención a la Diversidad dentro del marco de la escuela inclusiva, en un determinado centro educativo. Nuestra intención con este trabajo ha sido intentar obtener información en torno a la búsqueda de una educación que acoja a todo el alumnado, es decir, en torno a la escuela inclusiva. Hemos hecho este trabajo pensando en esa escuela que supone una superación de la lucha por la integración e implica que todos los niños reciban una formación independientemente de sus condiciones personales, sociales o culturales $\mathrm{e}$ independientemente de que presenten o no una discapacidad.
\end{abstract}

PALABRAS CLAVE: Inclusión. Derechos. Equidad. Desigualdad. Educación. Escuela inclusiva. Participación. Aprendizaje. Integración. Escuela integradora. Igualdad. Discapacidad. Comunidad educativa. Diversidad. Discriminaciones. Exclusión.

\section{Estado de la cuestión}

La inclusión hace relación a los cambios que la sociedad y la escuela necesitan realizar para que todos los individuos estén considerados en igualdad de derechos. La inclusión es una cuestión de derechos humanos, de equidad y de lucha contra la desigualdad, con el fin de que todo ciudadano reciba una educación acorde a sus características.

Por tanto, si por inclusión podemos entender el derecho a la participación y el aprendizaje, por escuela inclusiva entendemos aquella escuela que se construye sobre la participación y los acuerdos de todos los agentes educativos que en ella confluyen. En ella el proceso de aprendizaje del alumnado es la consecuencia de su inclusión en el centro escolar.

El origen del movimiento inclusivo se sitúa en los países anglosajones, dentro del marco de la educación especial. Concretamente se remonta a la ley de 1975 de los

\footnotetext{
${ }^{1}$ Alumno de Magisterio Lengua Extranjera. Universidade de Alcalá. Escola Universitária de Magisterio. Alcalá de Henares - Espanha. 28801 - labernia13@hotmail.com

${ }^{2}$ Alumno de Magisterio Lengua Extranjera Universidade de Alcalá. Escola Universitária de Magisterio. Alcalá de Henares - Espanha. 28801 - cokeortega@hotmail.com

${ }^{3}$ Universidad de Alcalá. Departamento de Didáctica. Alcalá de Henares - Espanha. 28801 -

eladio.sebastian@uah.es
} 
Estados Unidos (Education for All Handicapped Children Act), a través de la cual se consideraba que el alumnado con algún tipo de deficiencia podía recibir una educación apropiada en ambientes menos restrictivos (clase ordinaria). A partir de aquí surgen dos grandes movimientos: la iniciativa de educación regular (REI) y la escuela inclusiva. Si el primero es partícipe de integrar a los alumnos de educación especial en las clases ordinarias, el movimiento de la escuela inclusiva se centra en cómo aumentar la participación del alumnado con deficiencias en un aula ordinaria, independientemente de las características y niveles de cada persona.

La escuela inclusiva se opone a cualquier forma de segregación, de separación en el ejercicio de los derechos de la educación. Dicha escuela aparece como una alternativa a la Integración que ofrecía una educación de escasa calidad a los a.c.n.e.e. (alumnos con necesidades educativas especiales) o la más reciente denominación de a.c.n.a.e. (alumnos con necesidades de apoyo educativo) y adquiere la universalización a través de la Declaración de Salamanca de 1994 de Naciones Unidas. Al contrario que la Integración que se ocupaba principalmente del alumno con necesidades educativas especiales, la Inclusión centra su interés en todo el alumnado. Igualmente defiende la inclusión de todos los alumnos en aulas ordinarias independientemente de cuáles sean sus características personales.

En definitiva, se trata de lograr el reconocimiento del derecho que todos tienen tanto a ser reconocidos, como a reconocerse a sí mismos como miembros de la comunidad educativa a la que pertenecen, cualquiera que sea su medio social, su cultura de origen, su ideología, el sexo, la etnia o situaciones personales derivadas de una discapacidad física, intelectual, sensorial o de la sobredotación intelectual (SEBASTIÁN, 2007; ARNAIZ, 2003).

La educación inclusiva se asocia frecuentemente con la participación de los niños con discapacidad en la escuela común y de otros alumnos etiquetados "con necesidades educativas especiales". Sin embargo, esta acepción estaría más relacionada, según lo expresado anteriormente, con el concepto de integración educativa y no el de inclusión.

El concepto de educación inclusiva es más amplio que el de integración y parte de un supuesto distinto, porque está relacionado con la naturaleza misma de la educación regular y de la escuela común. La educación inclusiva implica que todos los niños y niñas de una determinada comunidad aprendan juntos independientemente de 
sus condiciones personales, sociales o culturales, incluidos aquellos que presentan una discapacidad. Se trata de un modelo de escuela en la que no existen "requisitos de entrada" ni mecanismos de selección o discriminación de ningún tipo, para hacer realmente efectivos los derechos a la educación, a la igualdad de oportunidades y a la participación.

El proceso de integración educativa ha tenido como preocupación central reconvertir la educación especial para apoyar la educación de los niños integrados a la escuela común, trasladando, en muchos casos, el enfoque individualizado, cfr Galve et al. (2002), y rehabilitador, propio de la educación especial, al contexto de la escuela regular. Desde esta perspectiva, se hacían ajustes y adaptaciones sólo para los alumnos etiquetados "como especiales" y no para otros alumnos de la escuela.

El enfoque de educación inclusiva, por el contrario, implica modificar substancialmente la estructura, funcionamiento y propuesta pedagógica de las escuelas para dar respuesta a las necesidades educativas de todos y cada uno de los niños y niñas, de forma que todos tengan éxito en su aprendizaje y participen en igualdad de condiciones y por ello se debe desarrollar un Plan de Atención a la Diversidad (PAD) específico para cada centro educativo. En la escuela inclusiva todos los alumnos se benefician de una enseñanza adaptada a sus necesidades y no sólo los que presentan necesidades educativas especiales.

Podemos establecer algunas de las diferencias entre integración e inclusión, como bien señalan Arnaiz (2003) y Moriña (2002).

\begin{tabular}{|c|c|}
\hline ESCUELA INTEGRADORA & ESCUELA INCLUSIVA \\
\hline Centrada en el diagnóstico & $\begin{array}{l}\begin{array}{l}\text { Centrada en la } \\
\text { colaboración. }\end{array} \\
\text { cesolución de problemas de } \\
\end{array}$ \\
\hline $\begin{array}{l}\text { Dirigida a la: Educación especial (alumnos con } \\
\text { n.e.e) }\end{array}$ & $\begin{array}{l}\text { Dirigida a la : Educación en general (todos los } \\
\text { alumnos) }\end{array}$ \\
\hline $\begin{array}{l}\begin{array}{l}\text { Basada en principios de igualdad y } \\
\text { competición }\end{array} \\
\end{array}$ & $\begin{array}{l}\text { Basada en principios de equidad, cooperación y } \\
\text { solidaridad (valoración de las diferencias como } \\
\text { oportunidad de enriquecimiento de la sociedad) }\end{array}$ \\
\hline La inserción es parcial y condicionada & La inserción es total e incondicional \\
\hline Exige transformaciones superficiales. & $\begin{array}{l}\text { Exige rupturas en los sistemas (transformaciones } \\
\text { profundas) }\end{array}$ \\
\hline $\begin{array}{l}\text { Se centra en el alumno (se ubica al alumno en } \\
\text { programas específicos) }\end{array}$ & Se centra en el aula (apoyo en el aula ordinaria). \\
\hline
\end{tabular}


aumentar la posibilidad de inserción.

Sebastián (2003) indica que para desarrollar un PAD es necesario hacer un análisis de las características y condiciones del contexto en el se va a poner en funcionamiento. De la misma manera que se tomarán como referencia, la indicación de este autor como veremos más adelante, los elementos que ha de tener, de forma teórica, el Plan de Atención a la Diversidad de un centro educativo.

Pensamos que indudablemente un documento como el PAD es esencial en la educación de hoy en día para prever una mayor y mejor integración del alumnado en la sociedad y el desarrollo de sus capacidades necesarias. Y es que gracias a este Plan se pretende dar una respuesta adaptada a las necesidades educativas especiales que presentan los alumnos. Este documento es bueno y de mucha utilidad porque nos ayuda a los docentes a mejorar la calidad de enseñanza de todos los alumnos y, más concretamente, la de aquellos con necesidades educativas especiales, asociadas a discapacidades motoras, sociales o culturales, de déficit cognitivo o de trastornos de conducta.

Bien es cierto que, tras haber comentado su gran utilidad en los centros educativos, ha de ser revisado debido a los continuos cambios de la sociedad en que vivimos, cambios tanto en el entorno del barrio, como en la tipología familiar, como en los alumnos. Y no sólo ha de estar pendiente de los cambios en la sociedad, sino también de las modificaciones legislativas o de la aparición de nuevas normas relacionadas con la diversidad y el alumnado con necesidades educativas especiales.

\section{Objetivo de la investigación}

El propósito de la investigación que hemos tenido a la hora de desarrollar este trabajo ha sido el de analizar el Plan de Atención a la Diversidad (en adelante PAD), desde el marco de la escuela inclusiva en el Colegio Público José Saramago.

\section{Instrumentos para desarrollar la investigación}

Para poder llevar a cabo nuestra investigación hemos desarrollado dos estrategias, de un lado la entrevista, para la que hemos contado con la ayuda inestimable 
de la Jefa de estudios del Colegio Público José Saramago, ubicado en Rivas Vaciamadrid (Madrid).

Por otro lado el análisis documental, para ello hemos leído, analizado y sintetizado el PAD del centro. Nuestro análisis queda de la siguiente manera.

\section{Análisis del plan de atención a la diversidad (PAD)}

Observamos el número de alumnos que en Primaria precisan atención, estando contemplados en el Plan de Atención a la Diversidad. Con la información recabada hicimos un estudio que reflejamos elaborando una tabla siguiente.

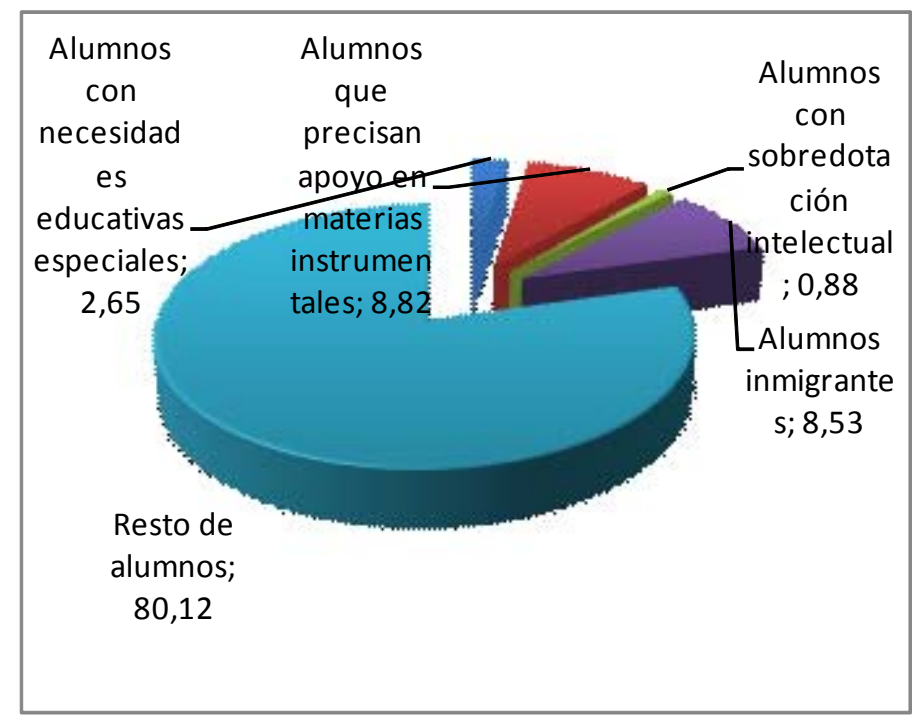

Si nos fijamos en la tabla, en el apartado de alumnos con necesidades educativas especiales, vemos que en Primaria el porcentaje de alumnos con estas características es realmente bajo. De un total de 340 alumnos, hay un 2,65\% que presentan necesidades educativas especiales. Con lo cual el porcentaje es mínimo.

Con respecto al número de alumnos que precisan apoyo en materias instrumentales (Lengua y Matemáticas) en Educación Primaria, vemos que es en el primer ciclo donde más alumnos requieren esa atención (hasta 15 alumnos) y que representan el $12,6 \%$, siendo en el tercer ciclo donde menos alumnos hay que precisen apoyo. Y con respecto al total de alumnos es cerca del $9 \%$ los alumnos que requieren ese apoyo. 
En cuanto a los alumnos superdotados, apenas encontramos niños con estas características, aproximándose al 1\% con respecto al total de alumnos en Primaria.

\begin{tabular}{|c|c|c|c|c|c|c|c|c|c|}
\hline \multicolumn{8}{|c|}{ COLEGIO PÚBLICO DE INFANTIL Y PRIMARIA JOSÉ SARAMAGO } \\
\hline \multicolumn{2}{|c|}{ NÚMERO TOTAL DE ALUMNOS EN PRIMARIA: 340 } \\
\hline CICLOS & TOTAL & $\begin{array}{c}\text { ALUMNOS } \\
\text { CON } \\
\text { NECESIDADES } \\
\text { EDUCATIVAS } \\
\text { de APOYO } \\
\text { EDUCATIVO }\end{array}$ & $\%$ & $\begin{array}{c}\text { ALUMNOS QUE } \\
\text { PRECISAN APOYO } \\
\text { EN MATERIAS } \\
\text { INSTRUMENTALS } \\
\text { (LENGUA Y } \\
\text { MATEM) }\end{array}$ & $\%$ & $\begin{array}{c}\text { ALUMNOS CON } \\
\text { SOBREDOTACIÓN } \\
\text { INTELECTUAL }\end{array}$ & $\%$ & ALUMNOS & $\%$ \\
INMIGRANTES & & & & \\
\hline $1^{\circ}$ & 119 & 3 & $2.53 \%$ & 15 & $12.07 \%$ & 2 & $1.68 \%$ & 14 & $1.18 \%$ \\
\hline $2^{\circ}$ & 123 & 4 & $3.25 \%$ & 9 & $7.31 \%$ & 1 & $0.81 \%$ & 5 & $4.06 \%$ \\
\hline $3^{\circ}$ & 98 & 2 & $2.04 \%$ & 6 & $6.12 \%$ & 0 & $0 \%$ & 10 & $10.2 \%$ \\
\hline TOTAL & 340 & 9 & $2.65 \%$ & 30 & $8.82 \%$ & 3 & $0.88 \%$ & 29 & $8.53 \%$ \\
\hline
\end{tabular}

Contemplando los alumnos inmigrantes de este centro, observamos que en el primer ciclo es donde más alumnos hay, representando cerca del 12\%. El total de alumnos inmigrantes en el colegio es de 29 alumnos, que representan el 8,53\% de niños matriculados en Primaria.

\section{Organización del PAD}

Lo primero que hemos querido conocer a la hora de hacer nuestro análisis son cuestiones introductorias tales como: qué es el PAD, quiénes lo elaboran, cuándo se elabora, cómo se revisa, etc.

\section{¿Qué es el PAD?}

El PAD es el conjunto de actuaciones organizativas, apoyos y refuerzos que un centro diseña y pone en práctica para que todo su alumnado tenga una respuesta ajustada a sus necesidades educativas generales y particulares.

¿Quiénes lo elaboran?

El documento lo elabora la Comisión de Coordinación Pedagógica (CCP), que está formada por el director del colegio, el jefe de estudios y los coordinadores de ciclo (un coordinador correspondiente a Educación infantil y uno por cada unos de los tres ciclos de Educación Primaria.), así como el Equipo de Orientación Educativa y Psicopedagógica, formado por el orientador, un especialista de pedagogía terapéutica y 
otro de audición y lenguaje. El profesor de audición y lenguaje es compartido con otro centro de la localidad.

\section{¿Cuándo se elabora?}

Se elabora una vez y permanece vigente hasta el momento en el que sean necesarias reformas en el mismo, ya sea debido a un cambio en la legislación educativa o por las cambiantes necesidades del conjunto de los alumnos o de cada uno de ellos, en particular.

El PAD del centro educativo está diseñado según las instrucciones del 19 de Julio de 2005 de la Dirección General de Centros Docentes de la Comunidad Autónoma de Madrid. Además está desarrollado atendiendo a tres puntos de vital importancia y que son:

- Desarrollo personal y social.

- Desarrollo cognitivo.

- Mejora de la convivencia.

¿Cómo se elabora y qué dificultades hay en su elaboración?

Su elaboración corre a cargo de los miembros mencionados anteriormente y con el consenso de todos ellos. Es aprobado por el Claustro de profesores e informado el Consejo Escolar, incluyéndose en la Programación General del Centro. Aunque técnicamente debería ser aprobado dentro del Proyecto Educativo por el Consejo Escolar.

En cuanto a las dificultades que se pudieron encontrar para su elaboración, básicamente fueron la adaptación a la normativa para todos los profesionales que participan en el PAD (se refiere a la adaptación a la sociedad actual) y los cambios en la terminología, para adaptarse a la legislación vigente y a las necesidades tan cambiantes de los niños. A pesar de ello, nos comentó la jefa de estudios que hubo un gran consenso, no añadiendo ninguna dificultad más a las anteriormente mencionadas.

¿Cómo y cada cuánto tiempo se revisa? 
Las posibles revisiones consisten en adaptarlo a los cambios en las legislaciones educativas de cada momento. Por lo demás, sólo se revisaría si alguno de los miembros participantes en su elaboración o en su puesta en práctica así lo demandase.

Tras el análisis que hicimos de los elementos anteriormente comentados, pasamos a analizar el contenido y la estructura del documento.

\section{Contenido y estructura del documento}

Partiendo de la base de que el PAD tiene un importante papel en las tareas diarias del colegio y resulta imprescindible para el buen funcionamiento del centro, la estructura del PAD del Colegio Público José Saramago es la siguiente:

1. Descripción del entorno y del tipo de alumnado del colegio.

2. Marco legislativo de referencia.

3. La atención a la diversidad. Qué se espera del Plan. Revisión del Plan.

4. Objetivos de este Plan.

5. Alumnado al que va dirigido.

6. Medidas de respuesta educativa.

7. Criterios para la atención del alumnado y propuesta de intervención.

8. Implicación de todos los profesionales.

9. Criterios de promoción.

10. Revisión del Plan.

11. Anexos.

Comparado el Plan de este centro educativo con el modelo que plantean otros autores como Sebastián (2003), observamos que ambos presentan una cierta similitud. El PAD del colegio estudiado está contemplado en el Proyecto Educativo de Centro (PEC). Dicho Plan consta de una introducción en la que se habla de la organización del centro; de las características de los alumnos y se dan a conocer nociones básicas sobre la atención a la diversidad y la descripción del entorno del centro educativo.

En cuanto al marco legislativo de referencia del PAD del centro educativo estudiado, es bastante amplio, pero podemos destacar en él referentes legislativos esenciales, tales como: 
- La Constitución Española de 1978, que establece el derecho a la educación de todas las personas.

- La LOE (2006), que regula la forma de plasmar ese derecho a la educación de todas las personas.

- La Ley Orgánica 9/1995, de 20 de Noviembre, de la participación, la evaluación y el Gobierno de los Centros Docentes.

Por tanto, se ve claramente que el PAD del centro educativo está adaptado a la normativa vigente, que no tiene otra finalidad que la de promover y facilitar una atención más individualizada que permita ajustar la ayuda pedagógica a las necesidades educativas del alumnado.

Hemos podido contemplar numerosos objetivos dentro del documento, entre los cuales vamos a destacar los siguientes:

- Ajustar la respuesta educativa a la realidad y necesidades de los alumnos del centro.

- Proporcionar al alumnado los recursos necesarios para lograr un mayor grado de desarrollo personal y social de éstos.

- Establecer colaboración entre los diferentes profesores que atienden a los a.c.n.e.e.

- Fomentar la participación de los padres en todos los temas relacionados con la educación de sus hijos y, en particular, con la escolarización de los mismos en este centro.

Podemos decir que los objetivos contemplados son suficientes puesto que básicamente han de comenzar por proporcionar al alumnado una respuesta educativa adecuada y de calidad, estableciendo los cauces de colaboración entre los distintos profesores que intervienen para que dicha respuesta sea adecuada al alumnado con n.e.e. y organizando los recursos personales y materiales del centro con el fin de desarrollar actividades encaminadas a la inserción y promoción de este alumnado.

En cuanto al alumnado al que va dirigido el PAD del centro educativo estudiado y comparado con el modelo de Sebastián (2003), observamos que en dicho PAD no está incluido el alumnado itinerante, el alumnado hospitalizado en largos períodos, el 
alumnado con discapacidad permanente y el alumnado con grave riesgo de abandono escolar y desmotivado.

Respecto a los criterios para la atención del alumnado y la propuesta de intervención, el Plan del centro educativo enumera los siguientes:

- Será prioritaria la atención a los a.c.n.a.e.

- Se priorizará la atención preventiva en Educación Infantil y en el primer ciclo de Educación primaria.

- Se priorizarán, además, los aspectos de autonomía personal y escolar.

- Las adaptaciones curriculares se realizarán para todos los a.c.n.a.e. En el caso de alumnos con bajo aprovechamiento del currículo en una o varias áreas y que su evaluación psicopedagógica no indique necesidades especiales, se realizarán las Adaptaciones Curriculares no significativas que considere el equipo educativo. Se podrán realizar también Adaptaciones Curriculares para grupo y Adaptaciones Curriculares del Proyecto Curricular según necesidades.

Pasando a otro apartado del PAD del centro, concretamente al de la implicación de todos los profesionales, el documento los divide en dos grandes grupos: los propios del centro educativo y los externos al centro educativo. Entre los primeros figuran:

- Tutores.

- Profesora de Pedagogía Terapéutica.

- Profesora especialista en Audición y Lenguaje.

- Equipo directivo.

Los externos al centro son:

- E.O.E.P.: como ya hemos comentado anteriormente en el análisis del documento, el centro cuenta con un orientador externo que acude un día a la semana. Se encarga de estudiar y diagnosticar niños con problemas de aprendizaje. El orientador se coordina con las profesoras de A. y L. y de P.T.

- La inspección, quien asesora, orienta y por supuesto apoya al centro educativo.

Creemos que la implicación de todos los profesionales comentados anteriormente está bien, aunque algo esencial entre todos ellos debe ser la coordinación. 
A nuestro juicio debe existir una coordinación de todo el equipo docente del centro, tanto interno como externo, puesto que todos ellos intervienen en el PAD. Con esa coordinación se pretende, sin duda, que conozcan y que pongan en marcha todas las actuaciones que se hayan planteado previamente. Bien es cierto que esa coordinación en algunos casos presenta dificultades.

También pensamos que es esencial dentro de este apartado la distribución de los espacios y la organización del aula mediante, por ejemplo, desdobles de grupos. En el centro educativo estudiado se llevan a cabo desdobles, siendo una práctica habitual para aquellos alumnos que requieren una atención más individualizada o alumnos con necesidades educativas especiales. Tuvimos la oportunidad, gracias a la jefa de estudios, de ver las clases donde se llevan a cabo dichos desdobles.

Pasando ya a analizar el contenido del PAD, vemos que existen dos tipos de medidas de respuesta educativa: ordinarias y extraordinarias, igual que en el modelo propuesto a seguir. Entre las medidas ordinarias contempladas en el modelo de referencia están:

- Adaptación del Proyecto Curricular de Etapa (revisión del proyecto, adaptación de las programaciones de área y adaptaciones curriculares no significativas).

- Planificación de los apoyos.

- Plan de refuerzo educativo.

Refiriéndonos al Plan de nuestro centro, hemos de decir que estas tres medidas están contempladas: los apoyos, los refuerzos y las adaptaciones del proyecto curricular de etapa que nuestro centro desarrolla, principalmente, a través de las adaptaciones en el currículum, en la metodología y en la acción tutorial. Tanto el refuerzo como el apoyo los imparten, preferentemente, el tutor del grupo que adapta en cada momento los contenidos y ejercicios dependiendo de las dificultades del niño, dentro del aula. El refuerzo y el apoyo están dirigidos a alumnos que presentan dificultades de aprendizaje en los aspectos básicos e instrumentales del currículo (área de Lengua y Matemáticas, principalmente). El número de alumnos que reciben estos apoyos son 15 en el primer ciclo de Primaria, 9 en el segundo ciclo y 6 en el tercer ciclo (ver anexo 1).

En cuanto a las adaptaciones curriculares no significativas, referidas a la secuenciación de objetivos, priorización de contenidos, planificación de actividades, 
adaptaciones metodológicas, procedimientos de evaluación..., las realiza el profesortutor y van encaminadas a alumnos que tienen dificultades para superar los objetivos propuestos. Para ello el docente selecciona contenidos básicos y unas actividades específicas o a veces seleccionando las que se aplican al grupo con carácter general. La metodología a seguir está basada en asignar algún período de tiempo de dedicación especial a ese alumno o bien reforzando los contenidos a estudiar utilizando materiales específicos gráficos, visuales o manipulativos. La evaluación se realizará mediante la revisión de actividades realizadas y el control de la asimilación por parte de los alumnos de los contenidos seleccionados.

En lo referente a los refuerzos educativos, este documento incluye los siguientes apartados: qué es un refuerzo educativo, a quién se dirige, dónde se realiza, las fases por las que pasa, informe de evaluación final del refuerzo.

Entre las medidas extraordinarias propuestas en el modelo de referencia figuran:

- Adaptaciones curriculares individuales significativas, a través del documento individual de adaptación curricular (D.I.A.C.) y que analizamos más tarde.

- Medidas educativas para alumnado con altas capacidades o sobredotación.

- Plan de atención para el alumnado que no promociona.

- Adaptaciones curriculares grupales.

En nuestro centro, las medidas extraordinarias propuestas son las adaptaciones curriculares y las medidas de flexibilización en la duración de la escolarización.

De las adaptaciones curriculares individuales significativas tenemos que decir lo siguiente: hay en total 9 alumnos en Primaria que presentan necesidades de apoyo educativo, de los cuales hay dos hiperactivos, cuatro con discapacidad intelectual y tres con dislexia. Estos alumnos necesitan un apoyo especial tanto en materias instrumentales (Matemáticas y Lengua), así como en Audición y Lenguaje (A. y L.). Son atendidos en otra aula por la especialista en A. y L. y también por la especialista en Pedagogía Terapéutica (P.T.). Ambas son titulares del centro. Para la realización de informes y estudios de casos, el centro cuenta con un orientador externo al centro que acude un solo día a la semana, concretamente los jueves. A nuestro juicio, el hecho de que el orientador acuda solamente un día a la semana implica que no se avance mucho en el estudio de niños que presentan problemas. De hecho nos comentaron en el centro que había poca coordinación entre el orientador y el resto del equipo docente. 
En cuanto a los alumnos superdotados, encontramos niños con estas características aproximándose al 1\% con respecto al total de alumnos de Primaria. El orientador ha sido el encargado de hacer un estudio de estos alumnos para detectar su sobredotación y, posteriormente, una adaptación curricular junto con el profesor-tutor, que se lleva a cabo en horario escolar, dentro de la clase. No existen en el centro estudiado alumnos con evaluación diagnóstica aunque sí en estudio.

Con respecto al alumnado que no promociona, este representa entre un 4\% ó 5\% del total de alumnos del centro. Los alumnos repetidores lo hacen al acabar el ciclo, pudiendo hacerlo una sola vez a lo largo de toda la etapa de Primaria. Los alumnos repetidores siguen el mismo programa que el resto de sus nuevos compañeros. A la hora de repetir un alumno, es el equipo educativo el que decide acerca de su promoción o no, informándole a la familia.

Por otro lado, observamos que no están contempladas en el centro las adaptaciones curriculares grupales.

Siguiendo de nuevo con el modelo de referencia, entre otras medidas figuran:

- Plan compensación educativa.

- Plan de atención para el alumnado con grave riesgo de abandono escolar y desmotivado.

En el centro estudiado hay un total de 29 alumnos inmigrantes, distribuidos de la siguiente manera: en primer ciclo, 14, en segundo ciclo, 5, y en tercer ciclo, 10. El centro dispone de un aula de enlace específica para alumnos extranjeros que llegan al centro con dificultades con el idioma, tanto en lectura como en escritura, principalmente. Estas aulas de enlace, aunque encuadradas en el Plan de Compensación educativa no es la respuesta más inclusiva, tendrán un tiempo máximo de permanencia de 6 meses y se llevarán a cabo, exclusivamente, para alumnos entre $2^{\circ}$ y $6^{\circ}$ de Primaria. Primeramente se les evalúa para ver su competencia curricular, estableciéndose un programa adaptado a ellos. El principal problema que se presenta son las pocas horas disponibles para que el profesorado atienda a estos alumnos. Hay que decir que presentan más dificultades en el lenguaje escrito, pues el lenguaje oral cuenta con mejores resultados al utilizarlo con mayor asiduidad. Conforme van avanzando estos alumnos se les va restando el número de horas de permanencia en esta aula, y se van integrando poco a poco en su nivel correspondiente. 
En cuanto a los criterios de promoción, ya hemos comentado anteriormente en el análisis del documento que es el equipo educativo, esto es, los docentes involucrados en la educación del alumno, el que decide acerca de la promoción o no de un alumno, informándole a la familia. En cuanto a los a.c.n.e.e., no promocionarán aquellos alumnos que presenten más de dos años de retraso curricular ni tampoco aquellos que manifiesten dificultades significativas en habilidades de competencia social. Creemos que los criterios de promoción son un aspecto importante dentro del PAD puesto que ellos mismos van a suponer el hecho de que el a.c.n.e.e. pase de un curso a otro superior, lo que conlleva que pase de una situación a otra con mejora, un avance en el proceso dinámico de aprendizaje dentro del contexto escolar.

Pasando al siguiente apartado del contenido del Plan, concretamente el de Revisión del Plan, hay que decir que este plan se implantó en el curso 2005/2006. Fue elaborado con la participación y el consenso de todo el equipo educativo y supuso un gran reto y un fuerte avance en la búsqueda de criterios de homogeneidad.

La Comisión de Coordinación Pedagógica juega un papel fundamental en la divulgación y aclaración de las cuestiones prácticas relativas a este plan para su conocimiento y uso por todos los profesionales del colegio.

Valoramos de manera positiva el hecho de que el PAD del centro educativo esté adaptado a la LOE, ley que ha impulsado la atención a la diversidad de los alumnos como uno de los principios básicos de la educación.

Como último apartado y contemplando el Plan se encuentran los anexos, que son los siguientes:

a) Plan de Acción Tutorial:

- En él se especifican las diferentes funciones que debe realizar el tutor en relación con su grupo autorizado.

- Incluye: objetivos, actividades, recursos, temporalización y ciclos en los que se implanta. Se diferencian estas funciones según se trate de la etapa de Educación Infantil o Primaria.

- Se realizará acción tutorial en base a diferentes grupos:

1. Tutor-alumno.

2. Tutor-grupo/clase.

3. Tutor-otros profesores.

4. Tutor-familias. 
b) Plan de Acogida: Hace una breve referencia a este plan (recogido en el documento que lleva su nombre), que se ideó para la mejor integración de los alumnos extranjeros procedentes de un sistema educativo diferente al sistema español.

Habiendo analizado y comentado la estructura del documento, podemos decir que el PAD tiene un buen contenido y una buena propuesta didáctica para la atención a la diversidad. Entendemos que si el PAD está diseñado y orientado a atender la diversidad del alumnado, el contenido de este documento en el centro educativo supera con creces estas expectativas, que no son otras que conocer qué necesidades tiene el alumnado y saber dar respuesta a esas necesidades de forma individualizada.

Creemos que el contenido del PAD del centro educativo está plenamente desarrollado para aportar al profesorado criterios, pautas e instrumentos para abordar la respuesta a la diversidad educativa.

Lo siguiente que hicimos fue realizar el análisis de la adaptación curricular, que pasamos a comentar a continuación.

Análisis de una adaptación curricular

La adaptación curricular individual (ACI) es el documento que concreta el currículo para un alumno determinado. La ACI debe ser realizada por un conjunto de profesionales y se recogerá en un documento individual de adaptación curricular (DIAC). Analizando el DIAC del colegio público José Saramago, vimos que constaba de las siguientes partes:

1. Datos de identificación del alumno.

2. Datos de identificación del documento.

a. Fecha de elaboración.

b. Personas implicadas en la realización de la adaptación y función que desempeñan.

c. Otros documentos del expediente del alumno.

3. Información sobre la historia personal del alumno.

a. Escolarización previa.

b. Aspectos clínicos más relevantes. 
c. Aspectos intelectuales más relevantes.

d. Aspectos comunicativos-lingüísticos.

e. Hábitos, autonomía y sociabilidad.

f. Previsión de servicios fuera del centro.

4. Datos relevantes para la toma de decisiones.

a. Estilo de aprendizaje y motivación para aprender (sus intereses por aprender y su manera de afrontar la actividad escolar).

b. Análisis del contexto escolar.

c. Contexto escolar y socio-familiar.

5. Propuesta de adaptación curricular (se especifica el tipo de adaptación que precisa el alumno).

6. Modalidad de apoyo (apoyos, tiempo, coordinación...).

7. Colaboración con la familia.

8. Criterios de promoción.

9. Seguimiento de la ACI.

Comparando la propuesta curricular adaptada de nuestro centro con el modelo teórico propuesto por Galve y Sebastián (2002), observamos que el esquema es básicamente similar. Partiendo de la base de que la propuesta curricular adaptada se efectúa principalmente sobre las áreas instrumentales (Lengua castellana - Literatura y Matemáticas), vemos que se diferencian dos grandes bloques:

1. Elementos de acceso.

2. Elementos básicos.

En cuanto a los elementos de acceso hay que distinguir entre elementos personales y elementos personales. Nuestro centro comparte lo expuesto por Galve et al (2002) en cuanto a la implicación de la familia en el centro, la existencia de especialistas en Audición y Lenguaje y de P.T., quienes actúan de forma coordinada. Además, los alumnos reciben de 4 horas de apoyo en materias instrumentales.

Con respecto a los elementos materiales, nuestro centro coincide con el modelo propuesto en cuanto a la existencia de espacios con material o mobiliario específico para realizar apoyo en lenguaje. Para el alumno con necesidades educativas especiales, el centro proporciona material específico como cuadernos de prelectura, libros hablados, tableros o tarjetas para la comunicación, cuadernillos de matemáticas, de ortografía, de 
lectura... Aunque el centro cuente con ordenadores en las aulas, no se hacen mucho uso de ellos. De la misma manera tampoco se utiliza material audiovisual frecuentemente.

Pasando al otro bloque, elementos básicos, hay que diferenciar cuatro puntos importantes:

- Objetivos

- Contenidos

- Metodología

- Evaluación.

El centro educativo diferencia entre objetivos a corto, medio y largo plazo. A largo plazo se incluye el ideal de persona, las aspiraciones de ese niño con n.e.e. Por ejemplo, que sea lo más autónomo posible o que encuentre un trabajo. A medio plazo, si por ejemplo se ha establecido la lectura comprensiva y la escritura funcional como objetivos básicos, pueden ser objetivos adecuados a corto plazo, que escriba su nombre o que lea carteles. Otra característica importante es que se personalicen los objetivos, por ello será necesario incluir objetivos distintos a los de sus compañeros (por ejemplo, habilidades de autonomía básica o sociales). Siempre se deben escoger objetivos prácticos, útiles, funcionales, aplicables y secuenciarlos en orden creciente de dificultad. Para seleccionar objetivos, el colegio sigue una serie de criterios que son los siguientes:

- $\quad$ Los más importantes y necesarios para el momento actual de la vida del niño, los que le sirven aquí y ahora.

- $\quad$ Los que tienen una mayor aplicación práctica en la vida social y los que se pueden aplicar a mayor número de situaciones.

- $\quad$ Los que sirven de base para futuras adquisiciones.

- $\quad$ Los que favorezcan el desarrollo de sus capacidades: atención, percepción, memoria, comprensión, expresión, autonomía, socialización.

En cuanto a los contenidos, se hace una selección de aquellos que son más adecuados enseñarles, estableciendo prioridades. Se ha de tener claro cuál es el objetivo educativo fundamental y preparar muchos y variados recursos para alcanzarlo. Por ejemplo, a la hora de enseñarle a escribir es esencial que aprenda el abecedario o el conocimiento de las letras mayúsculas y minúsculas. 
Por lo que a la metodología se refiere, el colegio coincide con el modelo de referencia en que trata de individualizar la metodología de trabajo con los alumnos con n.a.e. Para ello es esencial la coordinación entre todos los implicados en su educación: tutor, profesores de apoyo, familia... Se trata de utilizar estrategias que respondan a las características psicobiológicas del a.c.n.a.e., adaptándolas a su manera de pensar y actuar. Por ejemplo, si perciben mejor la información por la vía visual que por la auditiva, la presentación de imágenes, dibujos u objetos ayudará a mejorar su retención. Destacar también aspectos como actuar con flexibilidad, presentar actividades de corta duración, incluir actividades de refuerzo en la programación, planificar actividades variadas, aplicar programas de entrenamiento en habilidades sociales, establecer momentos en que se realicen ayudas mutuas entre iguales...

Tomando de nuevo como referente el modelo expuesto por Galve et al. (2002), la evaluación de nuestro centro coincide en que sea personalizada, adaptándola a las peculiaridades de cada alumno y realizándose en función de los objetivos planteados. La evaluación será continua. También serán importantes la observación y la revisión constante de las actuaciones. Se parte de una evaluación inicial para planificar las actuaciones educativas en función de esa evaluación. Se ha de procurar evaluar en positivo. Los sistemas de evaluación han de variarse, no siendo sinónimo de examen. Por ejemplo, visual y táctil en lugar de auditiva; oral y práctica en lugar de teórica y escrita; diaria en lugar de trimestral y basada en la observación en lugar de en exámenes; los que saben leer y escribir pueden realizar exámenes orales y escritos. Los resultados se recogerán en un boletín de notas que se les dará al igual que a los demás alumnos, en el que queden reflejados esos resultados y el grado en el que se van consiguiendo.

\section{Conclusiones}

Como conclusión queríamos comentar, a partir de la información levantada, entre otras cosas, que los alumnos con dificultades la mayoría del tiempo permanecen en el aula con el resto de los compañeros, siendo el profesor el que adapta o no en cada momento los contenidos y ejercicios, dependiendo de las dificultades del alumno. Aunque los alumnos que necesitan un apoyo especial en competencias básicas como Matemática, Lengua, Audición y Lenguaje, etc., se desdoblan trabajando fuera del aula 
con una especialista un número de horas a la semana, según las dificultades que tengan. Lo normal suelen ser tres o cuatro horas a la semana. En cuanto a los recursos, observamos las clases de apoyo y refuerzo en las que se realizan los desdobles y los apoyos, es decir comprobamos que se hacen fuera del aula.

También pudimos sacar como conclusión que se utilizan recursos como la biblioteca y una sala en la que guardan bastantes materiales, para ser utilizados en estas clases de apoyo para este alumnado.

Además el centro dispone de un aula de enlace específica. Esta aula está mayoritariamente dirigida a alumnos extranjeros recién llegados con dificultades con el idioma en lectura y escritura. Aunque la principal problemática que este tipo de alumnado presenta en el centro es que, con tan pocas horas, no pueden alcanzar el ritmo de la clase, sobre todo en el lenguaje escrito. Es cierto que se obtienen mejores resultados en el lenguaje oral, a nivel hablado, porque utilizan el castellano en su vida diaria. También pudimos sacar como conclusión que en la mayoría de los casos, se veía un gran avance desde Septiembre. Conforme van avanzando se les va restando el número de horas de atención individualizada o en pequeños grupos.

El centro dispone de un PAD bastante elaborado y coherente para la práctica educativa, aunque no podemos sacar conclusiones sobre su efectividad y real funcionamiento. Por otro lado observamos que existen adaptaciones curriculares realizadas en un documento base coordinado para todo el alumnado.

Existe una organización del profesorado para dar apoyo a los a.c.n.a.e. y además un esquema de coordinación, aunque parece mejorable a la vista de las observaciones y de la poca presencia del orientador en el centro.

Para finalizar queremos comentar que tras nuestra investigación podemos decir que la escuela inclusiva es altamente beneficiosa para todos, puesto que los chicos se vuelven capaces de demostrar sus mejores disposiciones ante sus compañeros con necesidades especiales, que también los necesitan a ellos, lo que puede considerarse una doble y elogiable perspectiva de educación moral y también cívica. Nosotros, como docentes, hemos de cuidar al máximo nuestra formación para conseguir que el triunfo de esa escuela inclusiva. 


\section{A STUDY ABOUT THE PROGRAM OF ATTENTION TO THE DIVERSITY IN A SPECIFIC EDUCATIVE CENTER}

ABSTRACT: This investigation gathers a study of the program of attention to the diversity within the frame of the inclusive school, in a specific educative center. Our intention with this work has been to try to obtain data around the search of an education of quality for all the pupils, that is to say, around the inclusive school. We have done this work thinking about that school that supposes an overcoming of the fight by integration and implies that all the children receive a formation independent of their personal conditions, social or cultural and independent of the fact that they present or not an incapacity.

KEYWORDS: Inclusion. Rights. Fairness. Inequality. Education. Inclusive school. Participation. Learning. Integration. Integrating school. Equality. Incapacity. Educative community. Diversity. Discriminations. Exclusion.

\section{REFERENCIAS}

ARNAIZ, P. Educación inclusiva: una escuela para todos. Málaga: Aljibe, 2003.

GALVE, J. L. et al. Adaptaciones curriculares: casos prácticos. Madrid: CEPE, 2002.

MORIÑA, A. I. Formándonos en la diversidad: hacia las prácticas colaborativas. Revista de Educación Especial, Málaga, n.32; p.39-54, 2002.

SEBASTIÁN, E. El plan de atención a la diversidad. En: MARTIN BRIS, M. Planificación educativa: intercambio de experiencias y perspectivas. Alcalá de Henares: Servicio Publicaciones UAH, 2003.

SEBASTIÁN, E. La atención a la diversidad en el contexto de la nueva sociedad. En: SEBASTIÁN, E.; MARTIN BRIS, M. (Coord.). Educación y sociedad global. Alcalá de Henares: UAH, 2007.

\section{BIBLIOGRAFIA CONSULTADA}

ESTEVE, F. M et al. La escuela inclusiva. Disponible en:

<http://www.uji.es/bin/publ/edicions/jfi11/9.pdf>. Acceso em: 25 mayo 2009.

GERENCIA REGIONAL DE EDUCACIÓN JUNÍN. Cultura escolar y escuela inclusiva. Disponible en: 
<http://www.drejunin.gob.pe/directorio/especial/CULTURA\%20ESCOLAR\%20Y\%20 ESCUELA\%20INCLUSIVA.pdf>. Acceso em: 25 mayo 2009.

ESCUELAS inclusivas: un paso más allá de la integración en los centros. Disponible en:

<http://www.laopiniondemalaga.es/secciones/noticia.jsp?pRef=2009052500_2_2615 Malaga-Escuelas-inclusivas-paso-alla-integracion-centros>. Acceso em: 25 mayo 2009. 